\title{
La carbamazepina antagonizza la soppressione di vitamina D-mediata dell'iperparatiroidismo secondario: patogenesi e possibilità terapeutiche
}

\author{
Adriana Dusso, Maria Vittoria Arcidiacono \\ Division of Experimental Nephrology, IRB Lleida, Lleida - Spain
}

\begin{abstract}
CARBAMAZEPIN ANTAGONIZES THE VITAMIN D-MEDIATED SUPPRESSION OF SECONDARY HYPERPARATHYROIDISM: PATHOGENESIS AND THERAPEUTICAL OPTIONS Abstract. This commentary updates: 1) the patophisiology responsible for carbamazepine-induced resistance to paricalcitol suppression of PTH in CKD; 2) the contribution of vitamin D deficiency to the adverse effects of carbamazepine; 3) the benefits of cholecalciferol supplementation either alone or in combination with paricalcitol in preventing/attenuating the onset of resistance to vitamin $D$ suppression of secondary hyperparathyroidism (SHPT); 4) the successful correction of vitamin D deficiency in chronic kidney disease (CKD) patients receiving anti-epileptic medication other than carbamazepine, with the goal of providing nephrologists with a practical, safe and efficacious therapeutic approach to improve outcomes in SHPT.
\end{abstract}

Key words: Vitamin D deficiency, Vitamin D metabolism, PTH, FGF23, Calcimimetics

Conflict of interest: None.

Financial support: None.

Accettato: 15 Settembre 2014

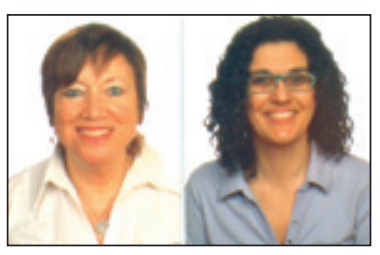

Dusso e Arcidiacono

Questo commento invitato si riferisce al caso di un paziente in cui è stata possibile la regressione di un grave stato di iperparatiroidismo secondario (IPTS) inizialmente resistente alla terapia con paracalcitolo (analogo del calcitriolo) e alla paratiroidectomia subtotale (7/8). La resistenza al paracalcitolo è risultata associata al prolungato trattamento col farmaco antiepilettico carbamazepina. Infatti, dopo l'accertamento di una grave carenza di vitamina D (livelli sierici di 25 -idrossivitamina $\mathrm{D}[25(\mathrm{OH}) \mathrm{D}]$ minori di $5 \mathrm{ng} / \mathrm{ml})$, è stato possibile ripristinare la sensibilità dell'IPTS alla terapia convenzionale (paracalcitolo combinato con calciomimetico) solo riducendo la posologia del farmaco antiepilettico contemporaneamente alla correzione della carenza di vitamina D con colecalciferolo.

L'obiettivo di questa discussione è quello di presentare ai nefrologi le attuali conoscenze riguardanti: 1) i meccanismi con cui la carbamazepina induce resistenza alla terapia con paracalcitolo in casi di IPTS; 2) il contributo della carenza di vitamina D all'aggravamento degli effetti avversi da carbamazepina; 3) i benefici della supplementazione di colecalciferolo, solo o in combinazione con paracalcitolo, nel prevenire/attenuare la resistenza dell'IPTS alla vitamina D in corso di CKD; 4) speciali considerazioni sulla correzione della carenza di vitamina $\mathrm{D}$ anche in pazienti trattati con farmaci antiepilettici diversi dalla carbamazepina; 5) un approccio terapeutico pratico, sicuro ed efficace per migliorare i risultati nell'IPTS.

In Figura 1 sono riassunti i principali meccanismi con cui la vitamina D sopprime il PTH a livello sia sistemico che paratiroideo e le possibili interferenze su questi da parte della carbamazepina. In individui con funzione renale normale, la forma inattiva della vitamina D3 (colecalciferolo) è trasformata nella sua forma ormonale attiva, 1,25-diidrossivitamina $\mathrm{D}([1,25(\mathrm{OH}) \mathrm{D}]$ o calcitriolo), attraverso due reazioni critiche successive di idrossilazione. La prima idrossilazione della vitamina $\mathrm{D}$ a formare $25(\mathrm{OH}) \mathrm{D}$ (o calcidiolo) è catalizzata da due citocromi P450, CYP27A1 e soprattutto CYP2R1, e si verifica principalmente (ma non esclusivamente) nel fegato. Poiché nell'organismo tutta la vitamina $\mathrm{D}$ circolante è convertita a $25(\mathrm{OH}) \mathrm{D}$ e quest'ultimo è più facilmente dosabile del colecalciferolo, la modalità corrente per stimare i livelli tissutali e circolanti di vitamina $\mathrm{D}$ in un individuo è proprio il dosaggio plasmatico del $25(\mathrm{OH}) \mathrm{D}(1,2)$. La seconda e cruciale idrossilazione per l'attivazione della vitamina $\mathrm{D}$ ha luogo principalmente (ma non esclusivamente) nel rene. La reazione è catalizzata da un altro citocromo P450, CYP27B1, 
Fig. 1 - Metabolismo sistemico e paraitroideo della vitamina $D$.

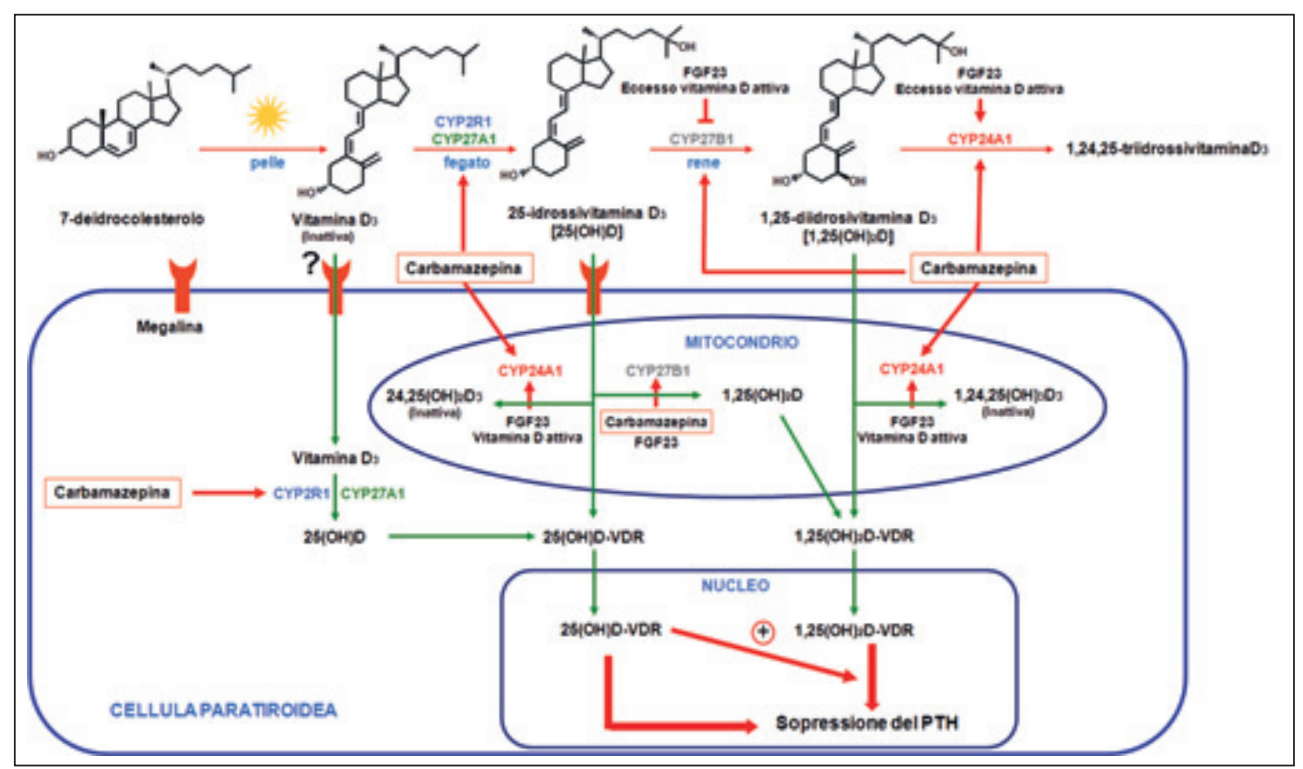

e produce calcitriolo, la forma attiva ormonale della vitamina D. A differenza della sintesi relativamente libera di $25(\mathrm{OH})$ $\mathrm{D}$, la produzione di calcitriolo è strettamente regolata per prevenire eventuali stati di ipercalcemia/iperfosfatemia causati dalla potente capacità del calcitriolo nel mobilizzare calcio e fosforo. La produzione renale di calcitriolo è soppressa dallo stesso calcitriolo (o analogo), quando i suoi livelli circolanti aumentano oltre il range di normalità, e dall'ormone fosfaturico FGF23.

Il calcitriolo è il più efficiente attivatore endogeno del recettore della vitamina D (VDR), che nelle paratiroidi è responsabile della soppressione del PTH $(1,2)$. Per questo motivo, per oltre trent'anni i nefrologi sono riusciti a sopprimere efficacemente l'IPTS nella CKD utilizzando calcitriolo o analoghi meno ipercalcemizzanti (per via orale o endovenosa) per compensarne la difettosa produzione da parte del rene danneggiato (2).

È stato recentemente dimostrato che anche nelle ghiandole paratiroidee avviene la conversione da vitamina $\mathrm{D}$ a $25(\mathrm{OH}) \mathrm{D}$ e da $25(\mathrm{OH}) \mathrm{D}$ a $1,25(\mathrm{OH}) \mathrm{D}$. Quest'ultimo (prodotto a livello intraghiandolare) attiva $\mathrm{i}$ VDR localmente, sopprimendo la sintesi di PTH con minimo o nullo impatto sui livelli sistemici di calcitriolo. L'attivazione paratiroidea della vitamina D a calcitriolo è particolarmente importante per la salute umana. Infatti nella popolazione generale, nonostante "normali livelli sierici di calcitriolo", la carenza di vitamina D (definita come livelli sierici di $25(\mathrm{OH}) \mathrm{D}<20 \mathrm{ng} / \mathrm{ml}$ ) è sufficiente ad aumentare la concentrazione sierica di PTH oltre i valori normali proprio per la difettosa produzione intraghiandolare di calcitriolo, necessario per la soppressione della sintesi del PTH.

Se nei soggetti con funzione renale normale la carenza di vitamina D causa un deficit di soppressione del PTH, questo effetto è assolutamente amplificato nei pazienti con CKD, condizione clinica caratterizzata da un deficit tanto di calcitriolo come di $25(\mathrm{OH}) \mathrm{D}$ già negli stadi più precoci e che si aggrava progressivamente nel corso della malattia. Infatti, è interessante notare che, diversamente dall'esperienza acqui- sita negli ultimi trent'anni nell'uso del calcitriolo, solo recentemente i nefrologi hanno riconosciuto il ruolo essenziale del rene nel mantenere i livelli circolanti normali di $25(\mathrm{OH}) \mathrm{D}$ e il contributo della carenza di vitamina D all'aggravamento dell'IPTS e alla sua resistenza alla terapia.

Nel rene normale il $25(\mathrm{OH}) \mathrm{D}$ è prima filtrato a livello glomerulare e successivamente riassorbito a livello tubulare. Il riassorbimento tubulare del $25(\mathrm{OH}) \mathrm{D}$ necessita di una proteina, megalina, che in corso di CKD viene persa rapidamente.

Questo deficit di megalina renale riduce la disponibilità del 25(OH)D non solo per la produzione renale del calcitriolo ma anche per mantenere i livelli sierici di $25(\mathrm{OH}) \mathrm{D}$ nel range necessario alla sintesi "non renale" di calcitriolo (come per esempio quella intraparatiroidea) (2). Va ribadito che la conversione tissutale "non renale" del $25(\mathrm{OH}) \mathrm{D}$ a calcitriolo sarebbe essenziale affinché gli individui sani mantengano tutti quei benefici di sopravvivenza associati ai livelli normali di vitamina $\mathrm{D}$. Inoltre, nella $\mathrm{CKD}$, è proprio la deficienza di vitamina D (misurata come $25(\mathrm{OH}) \mathrm{D}$ ) e non quella di calcitriolo, che si è dimostrata fattore di rischio indipendente di morte e di più severa progressione dell'insufficienza renale (2). Queste considerazioni danno maggior forza alle linee guida KDIGO che raccomandano la correzione di una carenza di vitamina D in tutte le fasi della CKD.

Un'appropriata correzione di un deficit di $25(\mathrm{OH}) \mathrm{D}$ nella CKD ha ulteriori vantaggi nel controllo dell'IPTS: in primo luogo il 25(OH)D può legarsi e attivare direttamente il VDR (3) anche senza essere convertito a calcitriolo; in secondo luogo è stato dimostrato che il $25(\mathrm{OH}) \mathrm{D}$ migliora l'efficacia del complesso calcitriolo/VDR (o paracalcitolo/VDR) nel sopprimere il PTH (4).

Poiché nella CKD la soppressione del PTH richiede adeguati livelli intracellulari di vitamina $\mathrm{D}$ e dei suoi metaboliti, è fondamentale evitare la degradazione del calcitriolo (o paracalcitolo) e del 25(OH)D sia a livello sistemico che della ghiandola 
paratiroidea. In tutti i tessuti vitamina D-mediata (comprese le paratiroidi) il principale responsabile della degradazione dei metaboliti attivi della vitamina D (calcitriolo, paracalcitolo) e del 25(OH)D è il citocromo CYP24A1, la cui espressione è aumentata da elevati livelli degli stessi metaboliti della vitamina $\mathrm{D}$ (il che riduce il rischio di raggiungere livelli tossici) (1) e anche dall'FGF23, che è precocemente aumentato in corso di CKD (e che, si ricorda, contemporaneamente inibisce il CYP27B1 renale) $(2,5)$. CYP24A1 renale ha una affinità 25 volte maggiore per il calcitriolo rispetto a quella per il $25(\mathrm{OH})$ $\mathrm{D}$ e ciò risulta particolarmente utile a ridurre il rischio di ipercalcemia perché il calcitriolo è molto più potente del $25(\mathrm{OH}) \mathrm{D}$ nell'attivare il VDR. Questa maggiore affinità del CYP24A1 renale per il calcitriolo, unitamente ai suoi livelli 1000 volte inferiori nella circolazione ( $\mathrm{pg} / \mathrm{ml}$ per il calcitriolo, $\mathrm{ng} / \mathrm{ml}$ per il $25(\mathrm{OH}) \mathrm{D})$ fa sì che il calcitriolo sia il primo metabolita a scomparire dalla circolazione.

La carbamazepina è un induttore di tutti i citocromi P450 $(6,7)$. Nel caso clinico in oggetto (paziente affetto da CKD) questa molteplicità di azioni può aver interferito con il metabolismo della vitamina D (e con la risposta dell'IPTS alla terapia somministrata) con meccanismi diversi, in grado di giungere a risultati diversi e addirittura contrari a seconda del prevalere dell'uno o dell'altro.

1) La carbamazepina induce il CYP24A1 renale che, come già descritto, è responsabile della degradazione di $25(\mathrm{OH})$ $\mathrm{D}$, calcitriolo e paracalcitolo. Nel caso clinico in oggetto, questo meccanismo da un lato può avere contribuito alla grave carenza di $25(\mathrm{OH}) \mathrm{D}$ (rilevati livelli inferiori a $5 \mathrm{ng}$ / $\mathrm{ml}$ ) e dall'altro aver causato una rapida degradazione del paracalcitolo fino a ridurne i livelli circolanti a una concentrazione inefficace a sopprimere il PTH.

2) La carbamazepina aumenta però anche il CYP27A1 e il CYP2R1, i due citocromi P450 che convertono il colecalciferolo a $25(\mathrm{OH}) \mathrm{D}$. Tuttavia, in questo paziente la verosimile preesistente deficienza di vitamina D (colecalciferolo) potrebbe aver reso questa strada meno efficiente per minore disponibilità di substrato mentre la successiva supplementazione di colecalciferolo avrebbe riattivato la via metabolica e in questo caso il farmaco antiepilettico avrebbe contribuito a correggere i livelli di $25(\mathrm{OH}) \mathrm{D}$, piuttosto che aggravarne il deficit.

3) Nel rene e nella paratiroide la carbamazepina può anche indurre il CYP27B1, responsabile della produzione di calcitriolo. Tuttavia, nella CKD, a livello renale questo potenziale beneficio è fortemente contrastato sia dalla limitata quantità di enzima sia da una potente inibizione del CYP27B1 causata dall'FGF23. Poiché, invece, nella paratiroide l'FGF23 induce (e non inibisce) il CYP27B1, in questa ghiandola la carbamazepina riuscirebbe a stimolare la sintesi di calcitriolo a patto che siano presenti normali livelli di substrato $25(\mathrm{OH}) \mathrm{D}$ anche in presenza di CKD.

In realtà non è ancora del tutto chiaro se la carbamazepina abbia una potenza superiore nell'attivare i citocromi agenti sulla degradazione della vitamina D o sulla sua attivazione. Comunque, la possibilità di effetti contrapposti sul metaboli- smo della vitamina $\mathrm{D}$ potrebbe fornire una spiegazione ai casi contradditori sull'impatto (positivo/negativo) della terapia con carbamazepina sulle ossa.

Deve poi essere tenuto presente che in questo paziente anche la supplementazione con colecalciferolo potrebbe aver contribuito a migliorare le anomalie del metabolismo della vitamina D (e la sensibilità dell'IPTS alla terapia) con meccanismi diversi.

1) La supplementazione con colecalciferolo ha contribuito certamente a correggere la grave deficienza preesistente e a riattivare la via metabolica verso i prodotti più attivi.

2) A differenza del $25(\mathrm{OH}) \mathrm{D}$, il colecalciferolo circolante non viene degradato dal CYP24A1 (la cui espressione è aumentata dalla carbamazepina) e, essendo anche più lipofilo, non necessita della megalina per entrare nelle cellule. Per queste caratteristiche la supplementazione con colecalciferolo può aver determinato una aumentata presenza della vitamina nel tessuto paratiroideo e contribuito, potenziando la conversione intraghiandolare a 25(OH)D e calcitriolo, a una maggiore soppressione del PTH.

Il miglioramento clinico di questo paziente potrebbe essere attribuito all'uso della terapia combinata di colecalciferolo e paracalcitolo. Infatti il calcitriolo (e il paracalcitolo) induce l'espressione della megalina (8), quindi teoricamente potrebbe aumentare i livelli intracellulari di $25(\mathrm{OH}) \mathrm{D}$ sia a livello renale che paratiroideo e di conseguenza, aumentare la produzione locale di calcitriolo. Non dimentichiamo infine che anche l'associato trattamento con cinacalcet ha sicuramente contribuito a controllare efficacemente l'IPTS, non solo perché il cinacalcet sopprime la sintesi del PTH, anche con bassi livelli di VDR e di CaSR, ma anche perché è in grado di aumentare i livelli di VDR diminuiti nella ghiandola paratiroidea (5), e quindi migliorare la risposta alla terapia combinata con colecalciferolo e paracalcitolo.

Riassumendo, il ripristino della sensibilità dell'IPTS al trattamento con paracalcitolo, ottenuto con successo in questo paziente grazie alla contemporanea somministrazione di colecalciferolo e paracalcitolo, potrebbe essere stato dovuto a diverse cause (Tab. I).

Indubbiamente, è impossibile per un medico valutare nella sua completezza l'asse della vitamina D e i suoi meccanismi di soppressione del PTH. Tuttavia, i risultati ottenuti in questo caso clinico suggeriscono l'utilizzo di un approccio terapeutico semplice consistente in un precoce utilizzo della vitamina $\mathrm{D}$, che potrebbe migliorare sia l'IPTS sia una sua resistenza alla terapia convenzionale. A tale proposito è raccomandabile valutare l'efficacia della supplementazione di colecalciferolo da solo o associato a basse dosi di vitamina D attiva - calcitriolo o paracalcitolo - misurando i livelli sierici di $25(\mathrm{OH}) \mathrm{D}$ al fine di mantenerli in range di normalità (superiori a $30 \mathrm{ng} / \mathrm{ml}$ ), come riportato nelle linee guida KDIGO, e dosando contemporaneamente i livelli sierici di PTH, che dovrebbero essere ridotti almeno del 30\%. Queste semplici ed accessibili misurazioni dell'efficacia di un trattamento con vitamina D nell'aumentare il $25(\mathrm{OH}) \mathrm{D}$ e contemporaneamente sopprimere il PTH sono fondamentali 
TABELLA I - PRINCIPALI CAUSE DELLA REGRESSIONE DELLA RESISTENZA DELL'IPTS AL TRATTAMENTO CON PARACALCITOLO

Miglioramento della captazione del $25(\mathrm{OH}) \mathrm{D}$ a livello della ghiandola paratiroidea

Azione sinergica del 25(OH)D con il ridotto paracalcitolo disponibile per l'attivazione del VDR paratiroideo e conseguente soppressione del PTH

Aumentati livelli paratiroidei di 25(OH)D per conversione locale del colecalciferolo

Ridotta degradazione del paracalcitolo sistemico e paratiroideo dovuta alla competizione degli aumentati livelli di 25(OH)D per il sito catalitico del CYP24A1 renale e paratiroideo

per personalizzare interventi (nutrizionali e/o con vitamina D attiva) atti a migliorare il controllo dell'IPTS, anche in presenza di un farmaco antiepilettico.

Si deve aggiungere che il successo della terapia con vitamina $\mathrm{D}$, è anche imprescindibilmente associato alla prevenzione di episodi di ipercalcemia ed iperfosfatemia. Pertanto è importante sottolineare che in questo paziente l'aggiunta di cinacalcet può aver permesso un aumento delle dosi di colecalciferolo e paracalcitolo senza elevare in modo significativo il rischio di ipercalcemia.

Rimangono due ultime considerazioni. Poiché la carbamazepina aumenta l'attività di CYP27A1 (6), le raccomandazioni delle linee guida KDIGO sull'utilizzo del colecalciferolo nella CKD (cioè di mantenere livelli normali di 25(OH)D sierico) avrebbero permesso in questo paziente un'aumentata conversione di colecalciferolo a $25(\mathrm{OH}) \mathrm{D}$ non solo a livello paratiroideo ma anche a livello di tutte le molteplici cellule che esprimono CYP27A1 e che contribuiscono a quel beneficio sulla sopravvivenza conferito, secondo Holick, da un normale stato di vitamina D indipendentemente da un efficace controllo dell'IPTS (9). Poiché l'iperfosforemia inibisce l'attività del VDR, la rigorosa prevenzione dell'aumento del fosforo sierico è tanto importante per l'efficacia della combinazione colecalciferolo/paracalcitolo quanto lo è la prevenzione di episodi ipercalcemici, ottenuta in questo paziente con la somministrazione di cinacalcet.

Nota importante: Qualora l'antiepilettico somministrato fosse, anziché induttore, soppressore dei citocromi P450, sarebbe del tutto inutile somministrare colecalciferolo. In tal caso la carenza di vitamina $\mathrm{D}$ dovrebbe essere corretta utilizzando il $25(\mathrm{OH}) \mathrm{D}$ piuttosto che il colecalciferolo perché la tappa compromessa sarebbe proprio l'attivazione della vitamina D da colecalciferolo a $25(\mathrm{OH}) \mathrm{D}$.

\section{Ringraziamenti}

Si ringrazia per la revisione critica e per i preziosi consigli ricevuti nella fase di traduzione del manoscritto il Dottor Stefano Michelassi della SOC di Nefrologia e Dialisi dell'Ospedale S.M. Annunziata, Azienda Sanitaria di Firenze.

\section{Riassunto}

Si presenta un aggiornamento su: 1) la fisiopatologia della resistenza indotta dalla carbamazepina all'azione soppressiva del paracalcitolo nell'iperparatiroidismo secondario (IPTS) a malattia renale cronica (CKD); 2) l'impatto di una deficienza di vitamina D sugli effetti indesiderati della carbamazepina; 3) i benefici della supplementazione con colecalciferolo - sia da solo che in associazione con il paracalcitolo - nella prevenzione/attenuazione della soppressione dell'IPTS vitamina D-mediata; 4) la correzione appropriata della carenza di vitamina $\mathrm{D}$ nei pazienti con CKD in terapia antiepilettica con carbamazepina, con lo scopo di fornire al nefrologo clinico un approccio terapeutico pratico, sicuro ed efficace, per migliorare l'outcome dell'IPTS.

Parole chiave: Deficienza della vitamina D, Metabolismo della vitamina D, PTH, FGF23, Calciomimetici

Dichiarazione di conflitto di interesse: Gli Autori dichiarano di non avere conflitto di interessi.

Contributi economici agli Autori: Gli Autori dichiarano di non avere ricevuto sponsorizzazioni economiche per la preparazione dell'articolo.

Indirizzo degli Autori:

Dr. Adriana Dusso, PhD

Experimental Nephrology Laboratory

IRB Lleida

Hospital Universitari Arnau de Vilanova, 1st floor

Avinguda Alcalde Rovira Roure 80

Lleida 25198, Spain

adusso@irblleida.cat 


\section{Bibliografia}

1. Dusso AS, Brown AJ, Slatopolsky E. Vitamin D. Am J Physiol Renal Physiol 2005; 289(1): F8-28.

2. Dusso AS, Tokumoto M. Defective renal maintenance of the vitamin D endocrine system impairs vitamin D renoprotection: a downward spiral in kidney disease. Kidney Int 2011; 79(7): 715-29.

3. Hoenderop JG, van der Kemp AW, Urben CM, et al. Effects of vitamin D compounds on renal and intestinal Ca2+ transport proteins in 25-hydroxyvitamin D3-1alpha-hydroxylase knockout mice. Kidney Int 2004; 66(3): 1082-9.

4. Lou YR, Molnar F, Perakyla M, et al. 25-Hydroxyvitamin $\mathrm{D}(3)$ is an agonistic vitamin $\mathrm{D}$ receptor ligand. J Steroid Biochem Mol Biol 2010; 118(3): 162-70.
5. Silver J, Rodriguez M, Slatopolsky E. FGF23 and PTH-double agents at the heart of CKD. Nephrol Dial Transplant 2012; 27(5): 1715-20

6. Samaniego EA, Sheth RD. Bone consequences of epilepsy and antiepileptic medications. Semin Pediatr Neurol 2007; 14(4): 196-200.

7. Mintzer S, Boppana P, Toguri J, et al. Vitamin D levels and bone turnover in epilepsy patients taking carbamazepine or oxcarbazepine. Epilepsia 2006; 47(3): 510-5.

8. Liu W, Yu WR, Carling T, et al. Regulation of gp330/megalin expression by vitamins A and D. Eur J Clin Invest 1998; 28(2): 100-7.

9. Holick MF. Vitamin D deficency. N Engl J Med 2007; 357: 266-81. 